\title{
On the origin of stroboscopic induced motion
}

\author{
BRUCE BRIDGEMAN and HENRY KLASSEN \\ University of California, Santa Cruz, California
}

\begin{abstract}
Apparent motion can be induced either by (1) relative motion between a target and a moving frame or (2) a tendency to judge the median plane to be biased toward the center of an asymmetrically positioned frame (the "Roelofs effect"): target position is then judged relative to the misplaced median plane. The first theory requires real motion; the second does not. We tested the two theories by abruptly displacing both a continuously visible target and a frame, asking subjects which of five possible positions the target occupied after the displacement. Rapid motions of target and frame simulated sensory events during saccadic eye movements in a structured visual field. Results with the continuously visible target were compared with results from a second condition identical to the first except that the projected stimuli were blanked for $1.0 \mathrm{sec}$ during the time of the jump. In a second experiment, the stimuli appeared in their offset positions with no transient. Subject behavior in the two experiments was identical, showing that the presence of a transient had no statistically significant effect. The Roelofs effect can account for our results, but relative motion cannot. The background frame offset perceptual judgments in these experiments, but would stabilize them under normal conditions.
\end{abstract}

In conventional experiments investigating induced motion, a frame moves laterally while a spot within it remains stationary with respect to both the observer and the world. Induced motion (Duncker, 1929) is defined as apparent motion of the spot. The motion might arise from either of two sources or from a combination of them; the first is the relative motion of the spot and the frame (as Duncker assumed), an exocentric, or object-relative, interpretation. A second possibility is that induction originates in the motion of the frame relative to the observer. In the latter case, the frame's motion would always cause its position to be asymmetrical with respect to the median plane of the observer's head at some point in the stimulus cycle, and the stimulus asymmetry might result in a realignment of the observer's apparent median plane in the direction of the center of the frame. This "Roelofs effect" (Roelofs, 1935) would, in turn, cause the spot in the induced motion display to appear to the opposite side of the median plane. This is also called an egocentric, or subject-relative, effect.

The original report of this effect was qualitative and sketchy, and much work remains to be done to completely characterize it. The effect was first described in a single paragraph of a larger paper on optical localization (Roelofs, 1935). The following is a translation of that paragraph in its entirety:

Another experiment is the following. A luminous rectangle is visible in an otherwise completely dark room. This rectangle can be moved in the frontal plane. One

This research was supported by NIH Grant EYO4137 to the first author. The authors' mailing address is: Department of Psychology, University of California, Santa Cruz, California 95064. can now try to bring either the right side or the left side of this rectangle into the apparent optical median plane. In the first case, the left half of the field of sight receives more light stimulation and probably also more motor impulses; in the latter case, the right half of the field of sight receives more light stimulation and probably stronger motor impulses. In fact the positioning of the right and left side was also unequal. The right side I adjusted somewhat more to the left, and the left somewhat more to the right.

The subject-relative explanation of induced motion does not invoke relative motion of the target and frame to explain the effect; only asymmetric stimulation is necessary. The explanation does require, however, that the subject have no access to information about eye position in the head; there is ample evidence that no such information is available (Bridgeman \& Stark,1981; Harris, 1974; Rock \& Halper, 1969) because subjects consistently misattribute stimulus offsets to deviations in the position of the eyes.

The two possible sources of the induced-motion phenomenon have been compared by Brosgole (1968). Using a mechanical spot-and-frame apparatus, he separated object-relative from subject-relative cues by changing instructions to the subjects while keeping exposure conditions constant. The result was that the amount of apparent motion a moving frame induces in an object is related to the extent to which it displaces the apparent straight-ahead location. Induced motion is always accompanied by apparent shifts of the apparent median plane. In a second experiment, Brosgole (1968) asked subjects to estimate the position of a static target when the frame was presented in a fixed off-center position. The apparent 
deviations of the target were as great as the deviations in the dynamic condition, showing again that induced "motion" effects could be explained by asymmetrical stimulation of the observer without recourse to relative-motion information.

Bacon, Gordon, and Schulman (1982) and Sugarman and Cohen (1968) performed similar experiments with a different response measure, finding a role for both object-relative and subject-relative effects. Subjects in these studies pointed to a target with an unseen pointer (open-loop pointing), which allowed access to a motor-oriented representation of visual space separate from the cognitive or focal representation of normal visual experience. Using the same measure, Bridgeman, Lewis, Heit, and Nagle (1979) showed that information about target position could be changed in the motor representation of visual space even though the change was masked from the cognitive system by saccadic suppression of displacement. In a further study, Bridgeman, Kirch, and Sperling (1981) used induced motion to cancel a signal in either the cognitive or the motor system, demonstrating that under the appropriate conditions information about target motion could enter the cognitive system without affecting the motor system, or vice versa. Thus, Sugarman and Cohen (1968) were measuring responses of the motor system, while Brosgole's measures accessed the cognitive system. Because the experiments reported below use a cognitive measure, the conditions compare most closely with those of Brosgole.

While Brosgole's results suggest a fundamental reinterpretation of induced motion in particular and of algorithms for spatial localization in general, three characteristics limit their generality. First, both Brosgole (1968) and Sugarman and Cohen (1968) used a pulley-driven mechanical apparatus that did not allow rapid stimulus displacements. Our goal was to apply the results to the problem of stabilization of the visual world across saccadic eye movements; thus, it was necessary to drive the stimuli at saccadic velocities. Duncker (1929) refers to this condition as stroboscopic induced motion; it differs from conventional induced motion in that displacement of the inducing frame is always visible. More recent analyses of subject-relative effects in induced motion have also been limited to slow continuous motion (Gogel, 1977; Nakayama \& Tyler, 1978; Wallach, O'Leary, \& McMahon, 1982).

Second, the most powerful and convincing test of the mechanism of induction effects would differentiate object-relative from subject-relative stimulation in terms of the stimuli themselves rather than Brosgole's (1968) instructions to the subjects. This can be accomplished by comparing the psychophysical responses to stroboscopic motion under three conditions: with the abrupt transient visible, with the transient occurring during a blank interval, and with asymmetrical stimulation without a motion transient.
Finally, it is methodologically desirable to measure subjects' judgments with modern forced-choice psychophysical techniques rather than subjective estimates of motion or position. This facilitates statistical analysis while reducing the effect of subject bias on the results.

\section{EXPERIMENT 1}

\section{Method}

\section{Apparatus}

Subjects sat in front of an opaque hemicylindrical screen with a radius of $58 \mathrm{~cm}$. Head position was stabilized with foreheadand chinrests. Vision was restricted by a horizontal baffle which blocked the lower region of the screen as well as the projection equipment. Chair height was adjustable to accommodate all subjects comfortably. The screen was uniformly illuminated at $32 \mathrm{~cd} / \mathrm{m}^{2}$. The stimuli were a dot of light and a rectangular frame, both with a brightness of $306 \mathrm{~cd} / \mathrm{m}^{2}$. The dot had a diameter of $0.35 \mathrm{deg}$, while the rectangle was $8.9 \mathrm{deg}$ tall $\times 15.2 \mathrm{deg}$ wide with a border $0.2 \mathrm{deg}$ in width. The stimuli were presented using two projectors with tungsten halogen lamps and infrared filters. A mirror galvanometer with a bandwidth of $0-65 \mathrm{~Hz}$ at $-3 \mathrm{~dB}$ interrupted each beam, so that the targets could be displaced. These were controlled by a PDP-11/23 computer. The mirrors were rotated using a third-order optimal control signal, which doubled the speed of mirror flips while eliminating overshoot.

\section{Procedure}

The subjects first viewed binocularly a slide which consisted of five evenly spaced dots 1.5 deg apart in a horizontal line, with the central dot located in the subjects' vertical median plane at eye level. They were informed that these dots were in the five possible locations to be occupied by the upcoming test stimulus. The subjects were then shown how to enter each location into the computer, using a key pad which had a row of five keys corresponding to the five stimulus positions. By holding one hand continuously over the keys, the subjects could easily enter their responses without having to look at the keys.

The subjects were then given a set of 15 practice trials. Each consisted of a dot appearing for $1 \mathrm{sec}$ in the center position, $1 \mathrm{sec}$ with no stimulus, then another $1 \mathrm{sec}$ with the stimulus reappearing in one of the five positions. The position was determined by a stored random number table in the computer. The subjects typed an estimate of target position and then pressed the "enter" key to advance to the next trial, which appeared after a delay of $5 \mathrm{sec}$. At the end of these trials, the subjects were informed of their performance. The 15-trial blocks were repeated until 100\% accuracy was achieved.

The response format was the same in the actual experiments. The subjects were informed that there would also be a frame projected onto the screen and that this frame might move, but responses should be entered as before, disregarding the frame. The subjects were told that mistakes entered could be corrected by the experimenter before advancing to the next trial.

The subjects were also told that they could rest between trials during the experiment and between experimental conditions, although they rarely opted to do so. Experiment 1 compared a control condition with a transient present to an experimental hidden transient condition.

Transient condition. During the transient trials, the dot appeared at the vertical center line of the screen, surrounded symmetrically by the frame. After an exposure of $1 \mathrm{sec}$, the dot and frame jumped simultaneously, but independently, to their respective positions and maintained this second configuration for $1 \mathrm{sec}$ before being extinguished (Figure 1, left). The shutters were not closed as the stimuli moved under mirror control from one position to the other. Thus, the motion was real rather than 
Control
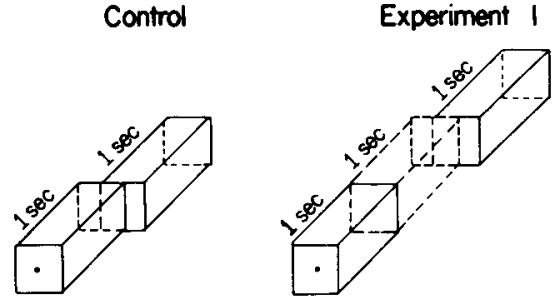

Figure 1. Stimulus location and timing in the three exposure conditions. Vertical and horizontal axes represent physical dimensions of the rectangle, while depth in the figure represents exposure duration. Displacements have been doubled for purposes of illustration.

apparent. For the dot, there were five possible positions, as described above. The frame, on the other hand, had three possible positions: either it could remain on center or it could move $4 \mathrm{deg}$ to the right or left. The displacement required about $8 \mathrm{msec}$. Thus, there were 15 trial types presented in random order. When the dot remained in its original position, the conditions were equivalent to the classical induced-motion stimulus.

Hidden transient condition. During the hidden transient trials, the dot and frame appeared for $1 \mathrm{sec}$ at the vertical center line, followed by a 1-sec blank period and then $1 \mathrm{sec}$ in the final position (Figure 1, center). Other stimulus parameters were the same as those in the transient condition. The Duncker exocentric motion theory predicts that induction should be less effective in this condition because no relative motion or displacement is present.

\section{Subjects}

There were eight subjects in Experiment 1, 4 males and 4, females. All were naive as to the purpose of the experiment. The experiment utilized a within-subjects design in which all subjects participated in both transient and hidden transient conditions.

\section{Results}

Contrary to the prediction of the exocentric theory, an ANOVA showed no significant difference between transient and hidden transient conditions (Figure 2a; Table 1). In addition, the transient/hiddentransient variable did not interact with frame position or dot position. This indicates that the transient condition has no greater effect than the hidden transient condition. In the latter condition, only the Roelofs effect can influence subjects' judgments. This result is shown by the similar positions of the two lines in Figure 2a.

The significant main effect for dot position simply indicates that when the dot was to the right of center subjects tended to judge its position to the right, and vice versa. Since the slopes of the lines in Figure 2a are less than 1, a range restriction is occurring. The significant main effect for frame position indicates that an offset frame position caused subjects to misjudge the dot in the opposite direction. This is shown by the separation of the lines for frame position in Figure 2b. Target and frame position interacted, indicating that the effect of the frame was dependent on dot position. There was a significant three-way interaction between dot position, frame position, and transient/hidden-transient condition, which can be attributed to the highly significant interaction of frame and target position. There were no significant subject effects.

When the spot remains in the center position without undergoing a displacement, the difference in indicated spot positions for the three frame positions is a measure of the experience of induced motion. This is the classic induced-motion stimulus situation. When the frame is moved, subjects perceive the spot to move in the opposite direction, even though it remains continuously visible and egocentrically fixed. Figure $2 c$ shows a "pinching" effect at position 3, suggesting that the effect is larger when a real spot displacement is added to the apparent one. The real displacement releases the stroboscopic induced motion, possibly by providing a real position transient which, once established, can be changed in apparent magnitude by induction effects. This result

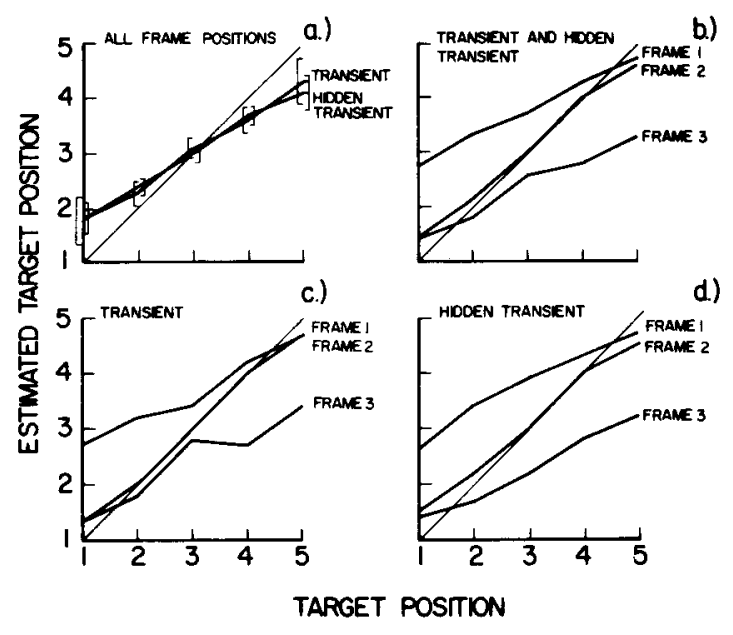

Figure 2. Subjects' estimates of target positions in Experiment 1: (a) separates transient trials from hidden-transient trials, summed across frame positions; the curves are nearly superimposed and are not significantly different. Brackets represent $1 \mathrm{SD}$ above and 1 SD below the mean. (b) shows the same trials separated by final frame position; (c) and (d) deplet the same data for the transient and the hidden-transient trials, respectively.

Table 1

Transient vs. Hidden Transient

\begin{tabular}{lccr}
\hline & F & df & p \\
\hline & Main Effects & & \\
Transient (T) & 0.05 & 1,7 & n.s. \\
Frame (F) & 2.043 & 2,14 & $<.001$ \\
Dot (D) & 85.95 & 4,28 & $<.001$ \\
& Interactions & \\
T X D & 1.38 & 4,28 & n.s. \\
T X F & 1.59 & 2,14 & n.s. \\
F X D & 14.79 & 8,56 & $<.001$ \\
T X F X D & 4.59 & 8,56 & $<.001$ \\
\hline
\end{tabular}


provides empirical support for the proposal by Bacon et al. (1982) that their induced motion effect is larger than that of Bridgeman et al. (1981) because the former study used a moving target. Both studies used open-loop pointing.

Experiment 1 showed no effects attributable to relative displacement. The design of the hidden transient condition controls for the center calibration and for the double stimulus exposure found in the transient condition, but introduces the possibility of a visual memory of the center position. If subjects could store target and frame position information during the 1-sec blanking interval, this information might be compared with the postblanking position to reproduce the effects of a physical transient. A control for this possibility is provided in Experiment 2.

\section{EXPERIMENT 2}

\section{Method}

\section{No-Transient Condition}

During the no-transient trials, the dot and frame appeared for $1 \mathrm{sec}$ at their final positions without a preceding exposure at the vertical center line (Figure 1, right). Thus, only a subject-relative Roelofs effect could change position judgments. Stimulus conditions were otherwise identical to those of Experiment 1.

\section{Subjects}

There were 11 subjects in Experiment 2, 5 male and 6 female psychology undergraduates who were naive as to the purpose of the experiment. The experiment utilized a within-subjects design with all subjects participating in both transient and no-transient conditions. Eight of these were subjects in Experiment 1.

\section{Results}

The ANOVA showed no significant difference between transient and no-transient conditions (Fig-

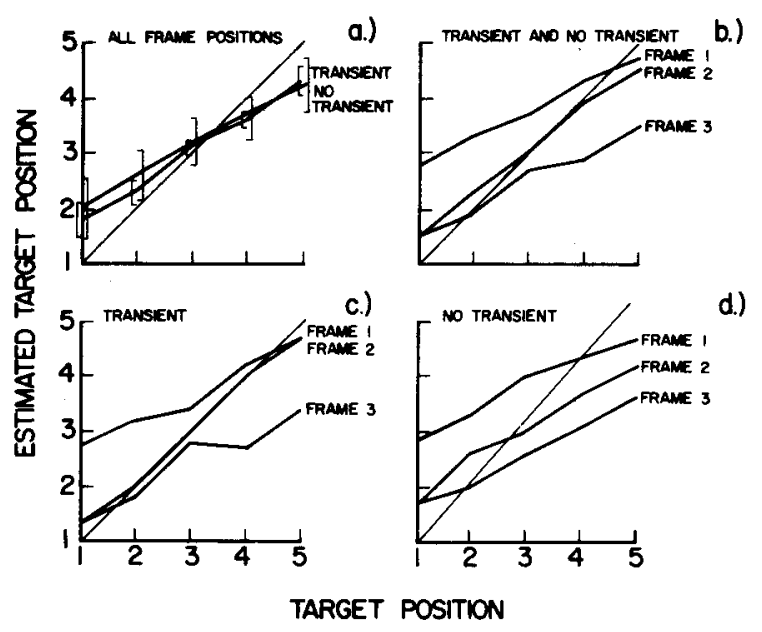

Figure 3. Subjects' estimates of target positions in Experiment 2. Display conventions are as in Figure 2.
Table 2

Transient vs. No Transient

\begin{tabular}{lccc}
\hline & F & df & p \\
\hline & Main Effects & & \\
Transient (T) & 1.88 & 1,10 & n.s. \\
Frame (F) & 20.83 & 2,20 & $<.001$ \\
Dot (D) & 168.47 & 4,40 & $<.001$ \\
\multicolumn{4}{c}{ Interactions } \\
T X D & 3.18 & 4,40 & $<.023$ \\
T X F & 0.17 & 2,20 & n.s. \\
F X D & 15.31 & 8,80 & $<.001$ \\
T X F X D & 8.22 & 8,80 & $<.001$ \\
\hline
\end{tabular}

ure 3a; Table 2), and the pattern of significances was identical to that of Experiment 1 except for a marginally significant interaction of transient/notransient condition with dot position. This latter effect seems to be due to the fact that no-transient trials did not provide subjects with an initial centrally located stimulus to serve as a reference for the straightahead. Thus, subjects tended to judge the dots to be further to one side. Our sample contained a majority of subjects who were biased to the right. There is also a slight, but consistent, increase in the standard deviation of position estimates in the no-transient condition, perhaps reflecting the lack of the centrally located stimulus.

The similarity of the results for the transient vs. no-transient experiment and the previous transient vs. hidden transient experiment does not substantiate the hypothesis that there is a visual memory for the center position either in accuracy or in pattern of responses.

\section{GENERAL DISCUSSION}

In these experiments, we have compared stroboscopic induced motion when both object-relative and subject-relative cues were present with induced motion when only subject-relative cues were available. We found that eliminating object-relative cues caused no reduction in induced motion, implying that the object-relative cues had made no contribution to the original stroboscopic induced-motion effect. This result extends the results of Brosgole (1968) to additional stimulus conditions and experimental designs. Others, however, have found very different contributions of object-relative and subject-relative effects in the mechanism of induced motion. Most of the experiments use one of five techniques for separating object-relative and subject-relative displacement.

(1) Subjects can be instructed to attend to either object-relative or subject-relative cues, as Brosgole did, with the result that the effect seems to be due to the subject-relative component.

(2) Object-relative cues depend on the perceived 
distance between the inducing frame and the test stimulus, while subject-relative information depends only upon visual angle. Gogel and Koslow (1971) have taken advantage of this difference between the eliciting requirements of the two kinds of reference systems to differentiate subject-relative from objectrelative effects in induced motion. By positioning the frame and the target at different distances from the subject, they showed that the amount of induced motion depends upon the angle through which the stimuli move rather than the real-world distance through which they move, with an added effect of adjacency, because a target and frame close to each other in space had a larger effect on one another than the same target and frame at greater separations. According to these authors, the result is consistent with a subject-relative hypothesis modified by the adjacency principle. This result is also in agreement with our results.

(3) Object-relative and subject-relative motion can be separated by eliminating the transient of motion, as we have done above, yielding results consistent with the supremacy of subject-relative effects.

(4) The effect of asymmetrical stimulation during induced motion can be eliminated by presenting multiple stimuli in the field of view, with symmetrically opposite induced motions visible simultaneously. Duncker (1929) showed that induced motion could be obtained in this condition by rotating a sectored disk and showing induced counterrotation of a concentric ring. The effect has been obtained for linear motions by Gogel (1977) and by Nakayama and Tyler (1978). Since subject-relative effects are cancelled in this condition, only the object-relative mechanism can mediate these examples of induced motion. This experiment does not rule out a contribution from subject-relative cues, however: By comparing a two-frame and two-disk configuration with a single-frame and -disk configuration, Gogel (1977) showed that the quantitative amount of induced motion was less when two oppositely moving figures were present simultaneously.

(5) The asymmetry of stimulation during lateral motion can be eliminated by moving a texture behind a fixed window so that the position of the window remains symmetrical while the texture undergoes unidirectional motion. Using this method, Wallach et al. (1982) found a significant induced-motion effect, but again it was smaller than the effect of a conventional moving frame with the same size and velocity. Because of ambiguities resulting from a visible fixation point, and from the cue given by the stationary edges of the window, Wallach et al. conclude that these experiments are ambiguous in their interpretation and that more research is necessary.

Wallach et al. (1982) considered the subject-relative explanation of induced motion to be untenable for three reasons. First, induced motion can be obtained without either asymmetrical stimulation or a moving window by using a moving pattern that fills the subject's visual field [although no evidence is given for this assertion, the literature on rotating drums (i.e., Brandt, Dichgans, \& Koenig, 1973) indicates that this is a strong effect]. Second, as Wallach et al. point out, the Roelofs effect shifts the apparent median plane by only a fraction of the total asymmetry of stimulation, although under some conditions induced motion can correspond to the full displacement of the surround. Wallach's final reason is the multiple display condition described in (4) above. All of these arguments show that subjectrelative effects cannot account for the entire phenomenon of induced motion under all conditions, but none of them eliminate the possibility that it is important under other conditions.

Our experiment differs from all of these in that rapid, rather than slow, motion was used. Rapid motion might have two opposite effects, one making object-relative cues stronger and the other emphasizing subject-relative cues. The first consideration is the interaction of visual and vestibular information in perceived motion. Slower background motions can induce feelings of self-motion (linear or circular vection) because the conflicting vestibular cue is available only at higher accelerations; the physics of the vestibular receptors makes them act as high-pass filters for motion. At higher stimulus velocities, including the velocities used here, the conflict with vestibular cues might reduce the magnitude of the subject-relative effect because vision specifies motion whereas the vestibular system does not. A second effect, one that favors subject-relative mechanisms in induced motion, is the duration of the stimulus. Since the motion in our experiment was nearly instantaneous, there was no time for normal motion-sensitive visual channels to be stimulated. Most visual cortex neurons that are sensitive to motion respond in a qualitatively different manner at saccadic velocities (Wurtz, 1969). Many of these cortical neurons are driven selectively by relative motion in the field rather than by homogeneous movement of the entire field (Bridgeman, 1972). Our data suggest that object relative cues are effective in perception only when this relative motion-sensitive system is stimulated.

In conclusion, the only way to reconcile the seemingly contradictory evidence about subject-relative vs. object-relative origins of induced motion is to hypothesize a nonlinear summation between the two kinds of effects. Thus, experiments which make possible stimulation by only one or the other system will both show effects, frequently in such a way that the object-relative plus the subject-relative effects, when measured in isolation, yield a larger total effect than is obtained when both mechanisms work together. 
We can now return to the question of the implications of these results for neurological algorithms of spatial orientation. The dominance of subjectrelative effects in stroboscopic induced motion implies an extension of visual capture to the case of perceptual stabilization of the visual world, for in our experiments the visual context (the rectangle) dominated vestibular and other cues in determining an offset of visual position. We can expect the same mechanism to function in normal conditions when the visual context is not displaced; normally, the visual conditions are the reverse of those presented in these experiments, since saccadic eye movements displace the optic array on the retina while the array itself remains egocentrically stable. This visual capture mechanism dominates corollary discharge when an optical array is present (Bridgeman \& Stark, 1981). Both in our experiments and in the normal condition, the position of a large peripheral array determines perceived locations; in the experiments, it offsets other judgments, whereas in the normal condition, it stabilizes them by taking advantage of the immobility of the visual array as a whole.

\section{REFERENCES}

Bacon, J., Gordon, A., \& Schulman, P. H. The effect of two types of induced-motion displays on perceived location of the induced target. Perception \& Psychophysics, 1982, 32, 353-359.

Brandt, Th., Dichoans, J., \& Koenno, E. Differential effects of central versus peripheral vision on egocentric and exocentric motion perception. Experimental Brain Research, 1973, 16, 476-491.

Bridgeman, B. Visual receptive fields sensitive to absolute and relative motion during tracking. Science, 1972, 178, 1106-1108.
Bridgeman, B., Kirch, M., \& Sperling, A. Segregation of cognitive and motor aspects of visual function using induced motion. Perception \& Psychophysics, 1981, 29, 336-342.

Bridgeman, B., Lewis, S., Heit, G., \& Nagle, M. Relation between cognitive and motor-oriented systems of visual position perception. Journal of Experimental Psychology: Human Perception and Performance, 1979, 5, 692-700.

Bridgeman, B., \& Stark, L. Efferent copy and visual direction. Investigative Ophthalmology and Visual Science Supplement, $1981,20,55$.

Brosgole, L. An analysis of induced motion. Acta Psychologica, $1968,28,1-44$.

Duncken, K. (1929) Über induzierte Bewegung. Psychologische Forschung, 1929, 12, 180-259.

GOGEL, W. (1977) Independent motion induction in separated portions of the visual field. Bulletin of the Psychonomic Society, $1977,10,408-410$.

Gogel, W., \& KosLow, M. The effect of perceived distance on induced movement. Perception \& Psychophysics, 1971, 10, 142-146.

HARR1s, C. S. Beware of the straight-ahead shift-a nonperceptual change in experiments on adaptation to displaced vision. Perception, 1974, 3, 461-476.

Nakayama, K., \& TYlen, C. W. Relative motion induced between stationary lines. Vision Research, 1978, 18, 1663-1668.

Rock, I., \& HALPER, F. Form perception without a retinal image. American Journal of Psychology, 1968, 82, 425-440.

Rozuofs, C. Optische Localisation. Archiv für Augenheilkunde, 1935, 109, 395-415.

Sugarman, R., \& Cohen, W. Perceived target displacement as a function of field movement and asymmetry. Perception \& Psychophysics, 1968, 3, 169-173.

Wallach, H., O'Leary, A., \& McMahon, M. Three stimuli for visual motion perception compared. Perception \& Psychophysics, 1982, 32, 1-6.

WURTz, R. Response of striate cortex neurons to stimuli during rapid eye movements in the monkey. Journal of Neurophysiology, 1969, 32, 975-985.

(Manuscript received November 22, 1982; revision accepted for publication March 21, 1983.) 